\title{
Identification and Control of Nonlinear Systems Using Multiple Models: Relay Feedback Approach
}

\author{
Yu-Chang Cheng and Cheng-Ching $\mathrm{Yu}^{1}$ \\ Department of Chemical Engineering National Taiwan University of Sci. and Technol., Taipei 106-07, TAIWAN
}

\begin{abstract}
In this work the relay feedback autotuning is extended to handle process nonlinearity. Local models from relay feedback tests are scheduled using the Takagi-Sugeno fuzzy modeling. The characteristics of the fuzzy implications are analyzed and an even simpler model is explored. The importance of the selection of the scheduled parameters is emphasized. One transfer function example and a recycle plant are used to illustrated the advantage of the simple model scheduling method. More importantly, the improved control performance can be achieved using already known process knowledge.
\end{abstract}

\section{Introduction}

Last decade has seen significant progress in the autotuning of PID controllers. Most of approaches are the variation of the Åström-Hägglund relay feedback tests (1984). First, a continuous cycling of the controlled variable is generated from a relay-feedback experiment and the important process information, ultimate gain $\left(K_{u}\right)$ and ultimate frequency $\left(\omega_{u}\right)$, can be extracted directly from the experiment. A controller can be designed according to $K_{u}$ and $\omega_{u}$ (e.g., Ziegler-Nichols method, Tyreus-Luyben Tuning, 1992; Shen and Yu, 1994). Applications of relay feedback based autotuners are shown throughout process industries.

Chemical processes are often operated at different steady-state. Changes in the operating condition are usually initiated by external factors. These parameters can be known a priori, , e.g., changes in the production rate or product specifications. The concept of multiple local models (Lainiotis, 1976; Narendra et al., 1995; Johansen and Foss, 1997; Banerjee et al., 1997) provides a new framework for automated chemical process control.

The relay-feedback based autotuning is proven reliable at the neighborhood of nominal operating point. Applications to complex chemical plants are also reported (Luyben et al., 1998; Yu, 1999). However, if the process is operated in a wide range of operating conditions

\footnotetext{
${ }^{1}$ to whom all correspondence should be addressed.

E-mail: ccyu@ch.ntust.edu.tw

Fax: +886-2-2737-6644
}

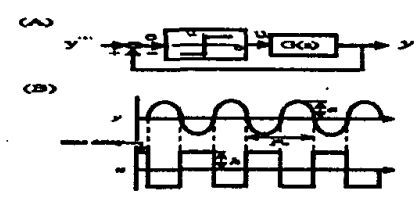

Figure 1: (A) Block diagram for a relay feedback system and (B) relay feedback test for a system with positive steady-state gain

the local controllers have to be detuned considerably (as the result of large uncertainty bound) in order to meet global robust stability. On the other hand, knowledge on process dynamics accumulated as the plant starts operation. Provided with an efficient autotuning procedure, multiple models can be obtained in a straightforward manner. Conventionally, these models are utilized via a look-up table type of approach.

Once multiple models are available, the next step is to employ the local model(s) at corresponding operating regime. In this work, fuzzy models of Takagi-Sugeno (1985) are used to schedule local models using linear membership function. Finally, a model scheduling procedure is proposed and the completeness of the global model varies with the available process knowledge. A transfer function example and a plantwide control example are used to illustrate the combined automatic tuning and model scheduling procedure.

\section{Autotuning}

Consider a relay feedback system where $G(s)$ is the process transfer function, $y$ is the controlled output, $y^{\text {set }}$ is the set point, $e$ is the error and $u$ is the manipulated input (Figure 1). Figure 1(B) illustrates how the relay feedback system works. A relay of magnitude $h$ is inserted in the feedback loop. Initially, the input $u$ is increased by $h$. As the output $y$ starts to increase (after a time delay $D$ ), the relay switches to the opposite position, $u=-h$. Since the phase lag is $-\pi$, a limit cycle with a period $P_{u}$ results (Figure 1 ). The period of the limit cycle is the ultimate period. Therefore, the ultimate frequency and ultimate gain are:

$$
\omega_{u}=\frac{2 \pi}{P_{u}} \quad \text { and } \quad K_{u}=\frac{4 h}{\pi a}
$$


where $h$ is the height of the relay and $a$ is the amplitude of oscillation. These two values can be used directly to find controller settings.

Based on the integrator plus time delay system, Tyreus and Luyben (1992) proposed a tuning rule which also utilizes the information of $K_{u}$ and $P_{u}$. Modifications are also proposed (Shen and Yu, 1994). For a PI controller, the settings are shown in Table 1.

Table 1 Different versions of the Ziegler-Nichols settings for PI controller.

\begin{tabular}{ccc}
\hline Rule & $K_{c}$ & $\tau_{I}$ \\
\hline original & $K_{u} / 2.2$ & $1.2 \cdot P_{u}$ \\
Tyreus-Luyben & $K_{u} / 3.22$ & $2.2 \cdot P_{u}$ \\
Shen-Yu & $K_{u} / 3$ & $2 \cdot P_{u}$ \\
\hline
\end{tabular}

The ultimate gain $\left(\hat{K}_{u}\right)$ ultimate frequency $\left(\hat{\omega}_{u}\right)$ can be used directly to back-calculate the local transfer function model. As pointed out by several authors (Tyreus and Luyben, 1992; Luyben and Luyben, 1997), the high frequency characteristic of the integrator plus time delay model offers an attractive means in modeling slow chemical processes. The transfer functions have the following form:

$$
G(s)=\frac{K_{p} \mathrm{e}^{-D s}}{s}
$$

The model parameters can be solved directly from the ultimate gain and ultimate frequency.

$$
K_{p}=\frac{2 \pi}{K_{u} P_{u}} \quad \text { and } \quad D=\frac{P_{u}}{4}
$$

The controller parameters of the modified ZieglerNichols tuning can be expressed explicitly in terms of $K_{p}$ and $D$.

\section{Model Scheduling}

Similar to gain scheduling, the model scheduling id defined as using different models as the operating condition changes. The process (or scheduled) variables $z$ is often referred to as model parameters or controller settings and the scheduling variables $x$ is the variable indicates the changes in the operating condition. They can often be set from process, e.g., production rate, product specification, process outputs etc. The model scheduling problem is then becomes: Given sets of process data $(z, x)$, find the functions $z=f(x)$ which can describe the global behavior.

The fuzzy modeling of Takagi and Sugeno (1985) is employed to construct the global model. The linguistic nature of the fuzzy set makes it easy to incorporate process knowledge into a quantitative model. A brief description of the fuzzy set is given. In fuzzy set, a variable $x$ may belong partially to a set. The membership function $(A)$ characterizes this degree of belonging. $A$ is defined as:

$$
A(x): x \rightarrow[0,1], \quad x \in X
$$

where $X$, generally, is a subset of $\mathcal{R}$ and the grade falls between 0 and 1 . In this work linear membership function is employed. The truth value $(T V)$ of a proposition " $x_{1}$ is $A_{1}$ and $x_{2}$ is $A_{2}$ " is expressed as: $A_{1}\left(x_{1}\right) \wedge A_{2}\left(x_{2}\right)=\min \left(A_{1}\left(x_{1}\right), A_{2}\left(x_{2}\right)\right)$ where $\wedge$ is the logical .AND. operator.

Takagi and Sugeno suggest that a multivariable system can be represented by the fuzzy implications $\left(R^{(j)}\right)$. Consider a multivariable system with $n$ input variables $\left(x_{i}, i=1, \cdots n\right)$ and an output $(z)$ with $k$ fuzzy implications. The $j^{\text {th }}$ implication is:

$$
\begin{array}{r}
R^{(j)}: \text { If } x_{1} \text { is } A_{1}^{(j)}, \cdots \text { and } x_{n} \text { is } A_{n}^{(j)}, \text { then } \\
z=p_{0}^{j}+p_{1}^{j} x_{1}+\cdots+p_{n}^{j} x_{n}
\end{array}
$$

Then, the output $z$ becomes:

$$
z=\sum_{j=1}^{k} \beta_{j}\left(p_{0}^{j}+p_{1}^{j} x_{1}+\cdots+p_{n}^{j} x_{n}\right)
$$

where

$$
\beta_{j}=\frac{A_{1}^{(j)}\left(x_{1}\right) \wedge \cdots \wedge A_{n}^{(j)}\left(x_{n}\right)}{\sum_{j=1}^{k}\left[A_{1}^{(j)}\left(x_{1}\right) \wedge \cdots \wedge A_{n}^{(j)}\left(x_{n}\right)\right]}
$$

The Takagi-Sugeno method offers a general framework to establish a nonlinear (global) model between the scheduling variable $x$ (e.g., production rate, product specification etc.) and the process variable $z$ (e.g., process steady-state gain, time constants, time delay etc.). Let us use a single-input-single-output example to analyze the fuzzy model.

Example 1. Suppose the trend of the process variable $(z)$ around two operating points are known. We have the following two implications:

$$
\begin{array}{r}
R^{(1)}: \text { If } x \text { is } A^{(1)} \text { then } z=x+1 \\
R^{(2)}: \text { If } x \text { is } A^{(2)} \text { then } z=0.5 x+0.5
\end{array}
$$

The membership functions $A^{(1)}$ and $A^{(2)}$ are given in Fig. 2. If the scheduling variable $x=1.8$, the two implications give: $z=2.8$ with a truth value of $T V=0.8$ and $z=1.4$ with $T V=0.2$. Thus, the process variable inferred by $R^{(1)}$ and $R^{(2)}$ is $z=2.52$ Figure 3 shows that the fuzzy modeling results in a piecewise nonlinear function between $z$ and $x$. Actually, the nonlinear function can be found analytically. Obviously, a linear combination of two linear functions (Fig. 3) is used for the fuzzy reasoning. That results into a nonlinear 
function. $\square$ Several observations can be made immediately. Consider the linear membership functions in Fig. 2 where the scheduling variable $(x)$ superimposes the same range.

O1. If the output variable $z$ shows the same trend as the scheduling variable $x$ varies (i.e., the slopes have the same sign), then the resultant nonlinear function is monotonic.

O2. If the output variable $z$ shows different trends as the scheduling variable $x$ varies (i.e., the slopes have different signs), then the resultant nonlinear function is non-monotonic.

An even simpler model scheduling mechanism can be devised.

Example 2. Suppose the trend of the process variable is not known. The two data points we have are: $z=3$ at $x=2$ and $z=1$ at $x=1$. The implications of Example 1 then becomes:

$$
\begin{array}{ll}
R^{(1)}: & \text { If } x \text { is } A^{(1)} \text { then } z=3 \\
R^{(2)}: & \text { If } x \text { is } A^{(2)} \text { then } z=1
\end{array}
$$

The membership functions $A^{(1)}$ and $A^{(2)}$ are the same as those in Fig. 2. Again, for the case of $x=1.8$, the inferred process variable becomes $z=2.52$. For this system (process with monotonic trend), the results of both examples are practically the same. Actually, the resulting function is simply a linear interpolation.

The following observation points out its limitation.

03. If the trend of the output variable $z$ is not included, then the resultant function is simply linear interpolation of these two different data points which is always a monotonic function.

Despite its limitation, this simple approach offers an attractive alternative in most cases.

Another nice feature of the Takagi-Sugeno modeling is that once a new identification result becomes available, we can simply add another implication to the original sets (e.g., $R^{(1)}$ and $R^{(2)}$ in Example 2. For example, if we obtain a new data $x=1.5$ and $z=2.2$, the third implication becomes: $R^{(3)}$. If $x$ is $A^{(1)}$ then $z=2.2$ Notice that the ranges of the input variable $(x)$ in the membership function should be modified accordingly. (Certainly, it can be done in a automated manner.) Figure 4 shows the modified membership functions.

¿From previous discussion, it should be recognized that nonmonotonic behavior is more difficult to capture. It generally requires more process information in quantity as well as in quality. Therefore, in building a global model, it is important to select appropriate scheduled variables $(z)$ such that the nonmonotonic behavior can be avoided. Let use the linear integrator plus time delay model to illustrate the effect of different scheduled variables. Suppose the T-L tuning (Table 1 ) is employed to tune the typical slow processes (Eq. 3).

Consider the first case where both model parameters $\left(K_{p}\right.$ and $\left.D\right)$ increase as the operating condition changes (i.e., increase in the scheduling variable). Figure $5 \mathrm{~A}$ shows that the controller parameters also changes monotonically as the operating condition varies. But a better global model can be achieved if the model parameters are selected as the scheduled variables. The second case is that the $K_{\mathrm{p}}$ and $D$ move toward different directions as the operating condition changes (Fig. $5 \mathrm{~B})$. This is a more likely situation in process systems. However, if the controller parameters are used as the output variable, we have a nonmonotonic behavior in the controller gain $K_{c}$ as shown in Fig. 5B. As mentioned earlier, we need either more identification results or very precise description of the process trend to find a reasonable global model. The examples clearly illustrate the importance in selecting the scheduled variables.

\section{Results and Discussion}

For the practicality and simplicity, the local model is described by the integrator plus time delay system (Eq. 3 ). The controller tuning of Shen and Yu gives a gain margin of $2.83(G M=2.83)$ and phase margin of 46.1 degree $\left(P M=46.1^{\circ}\right)$ for all possible model parameters (i.e., $K_{p} \neq 0$ and $D \neq 0$ ). First we would like to know how well the nominal controller settings work. Considering the nominal condition of $\bar{K}_{p}=1$ and $\bar{D}=$ 1, Figure 6 shows the region of robust stability (RS). For example, the closed-loop system becomes unstable when $K_{p}^{\prime}=2$ and $D=2$ (Fig. 6) and it remains stable for small values of $K_{p}$ and $D$. Figure 6 shows that the settings remain stable for a fairly large region in the parameter space. A more useful assessment is the region can achieve the robust performance (RP). In this work, a very simple measure of the RP is defined: A system is RP if and only if $2.21 \leq G M \leq 3.95$ and $36.1^{\circ} \leq P M \leq 56.1^{\circ}$. It means we allow the $1 / G M$ and $P M$ to vary by \pm 0.1 and $\pm 10^{\circ}$, respectively. The region of the RP can then be found by solving equations describing $G M$ and $P M$. The shaded area in Fig, 6 indicates the parameter space where the RP can be achieved. In other words if the process drifts out of the shaded area the controller has to be retuned for good performance. Therefore, the region of the RP can be used to evaluate the effectiveness of model scheduling approaches.

Suppose the process are operated at three different conditions: high, nominal and low productions which correspond to $K_{p}=D=0.5,1$ and2, respectively (indicated by $x$ in Fig. 7). We examined three approaches: (1) fixed gain control, (2) crisp switching control and (3) fuzzy switching control. By crisp switching, 
we mean the model parameters (and consequently the controller parameters) is chosen from one of the three set if certain condition in the scheduling variable is met. the fuzzy switching implies the model parameters (and consequently the controller parameters) is generated from a fuzzy model (e.g., $R(j)$ in Example 1 . In the fixed gain control, we only have the nominal settings, the region of RP is indicated by the shaded area in the middle (Fig.-.7):- If we decide to use the crisp model switching among three sets of model parameters, then, at best, the regions of RP are the three shaded areas. However, if the local models are scheduled according to the Takagi-Sugeno fuzzy implications, we have a much larger region for the RP as shown in Fig. 7. The degree of sophistication in the consequence (e.g., with or without knowledge on process trend) has little effect on the RP region.

Example 3. Consider the following nonlinear system:

$$
y=\frac{K_{p}(y) \mathrm{e}^{-D(y) s}}{s} u
$$

with $K_{p}(y)=D(y)=y+1$. Nominally the system is operated at $y=0$. A PI controller with the T-L tuning is employed and the results shows that the fixed gain control gives oscillatory set point responses (dashed line in Fig. 8) If we obtain new identification results at $y=1$, a fuzzy model scheduling can be constructed. The results show that much better set responses can be obtained (solid line in Fig. 8) when these two local models are scheduled using the simple Takagi-Sugeno fuzzy implications.

The second example is a reactor/separator plant studied by Yu (1999). The conventional control structure is designed and the nominal controllers parameters are tuned using the sequential tuning approach of Shen-Yu (Shen and Yu, 1994). The production rate is used as the scheduling variable. The nominal value is $460 \mathrm{lbmol} / \mathrm{hr}$ and as the economic condition changes the plant produces $70 \% \sim 130 \%$ of the nominal rate.

If only the nominal model parameters are available, we use the settings at all possible operating points. The dashed lines in Fig. 9 show the closed-loop responses for $\pm 30 \%$ changes in the production rate. On the other hand, as the process knowledge accumulates, we have the model parameters at $+20 \%$ and $-30 \%$ of the nominal production. The fuzzy modeling can, then, be employed for the model scheduling. The integrator plus time delay model is appropriate for this application. Again, the simplest fuzzy modeling is used: Figure 9 shows that improved closed-loop responses can be obtained using the model scheduling. Notice that for the entire range of the production $(-40 \% \sim+40 \%)$, the model parameters show slight nonmonotonic behavior.

\section{Conclusion}

In this work the relay feedback autotuning is extended to handle process nonlinearity. Local models from relay feedback tests are scheduled using the TakagiSugeno fuzzy modeling. The characteristics of the fuzzy implications are analyzed and an even simpler model is explored. The importance of the selection of the scheduled parameters is emphasized. One transfer function example and a recycle plant are used to illustrated the advantage of the simple model scheduling method. Simulation results show that improved transient responses and (unknown) disturbance rejection can be achieved when additional local models becomes available. More importantly, the controller design and model scheduling procedure is carried out in an automated manner.

\section{References}

Åström, K. J., and T. Hägglund, "Automatic Tuning of Simple Regulators with Specifications on Phase and Amplitude Margins," Automatica 20, 645 (1984).

Banerjee, A., Y. Arkun, Ogunnaike, B., and Pearson, R., "Estimation of Nonlinear Systems Using Linear Multiple Models," AIChE J. 43, 1204 (1997).

Johansen, T. A., and B. A. Foss, "Operating Regime Based Process Modeling and Identification," Comput. Chem. Eng. 21, 159 (1997).

Lainiotis, D. G., "Partitioning: A Unifying Framework for Adaptive Systems: Estimation," Proc. IEEE 64, 1126 (1976).

Luyben, W. L., and M. L. Luyben Essentials of Process Control; McGraw-Hill: New York, 1997.

Luyben, W. L., B. D. Tyreus, and M. L. Luyben Plantwide Process Control; McGraw-Hill: New York, 1998.

Narendra, K. S., J. Balakrishnan, and M. K. Ciliz, "Adaptation and Learning Using Multiple Models, Switching and Tuning" Control Syst. Mag., 15 (June), 37 (1995).

Shen, S. H., and C. C. Yu, "Use of Relay-Feedback Test for the Automatic Tuning of Multivariable Systems," AIChE J. 40, 627 (1994).

Takagi, T., and M. Sugeno, "Fuzzy Identification of Systems and Its Applications to Modeling and Control," IEEE Trans. Syst. Man Cyber. SMC-15, 116 (1985).

Tyreus, B. D., and W. L. Luyben, "Tuning of PI Controllers for Integrator/Deadtime Processes," Ind. Eng. Chem. Res. 31, 2625 (1992).

Yu, C. C. Autotuning of PID Controller: Relay Feedback Approach; Springer-Verlag: London, 1999. 


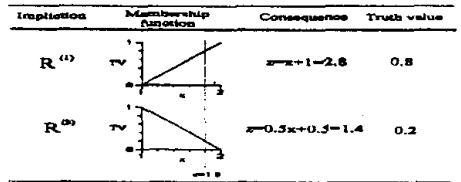

Figure 2: Fuzzy implications of Takagi-Sugeno (Example 1).

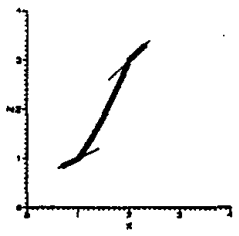

Figure 3: The resultant global model from fuzzy modeling (Example 1).

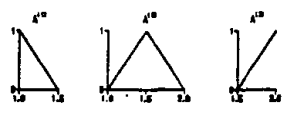

Figure 4: The linear membership functions for the case of three intervals in input variable.
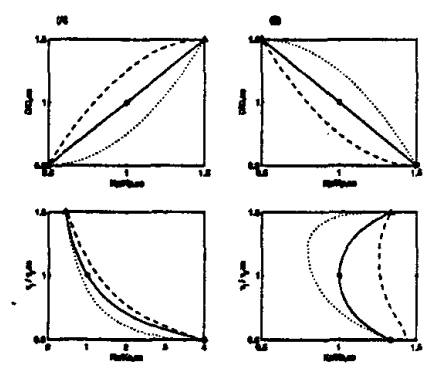

Figure 5: The effect of the selected scheduled (output) variables.

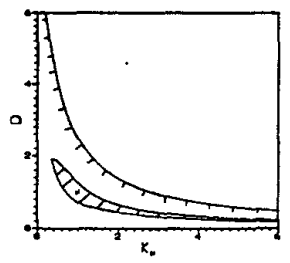

Figure 6: The regime of robust stability (RS) and robust performance( $R P$, shaded area).

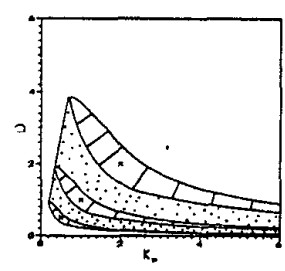

Figure 7: The regimes of robust performance at different operating conditions (indiated by $x$ ) for the fixed gain control (the middle shaded area), crisp switching (shaded areas) and fuzzy switching.

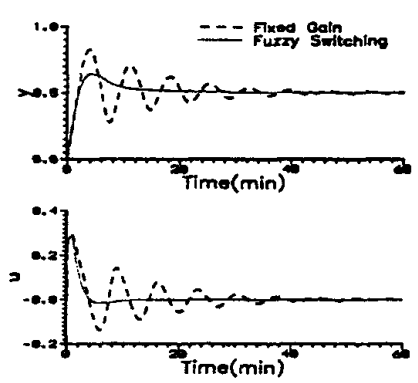

Figure 8: Set point responses of Example 3 using the fixed gain control and fuzzy switching.
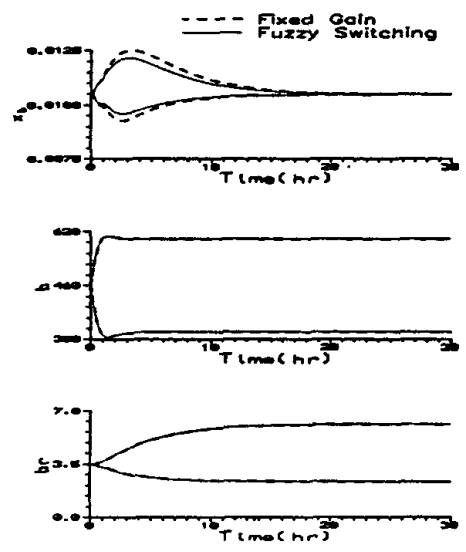

Figure 9: Load responses of the recycle plant for $\pm 30 \%$ production rate changes using the fixed gain control and fuzzy switching. 